\title{
Surface Cooling Rapidly Induces Coordinated Activity in the Upper and Lower Airway Muscles of the Fetal Lamb in Utero
}

\author{
BARBARA M. JOHNSTON, TANIA R. GUNN, AND P. D. GLUCKMAN \\ Developmental Physiology Laboratory, Department of Paediatrics, School of Medicine, University of Auckland, \\ Auckland, New Zealand
}

\begin{abstract}
Stimulation of cutaneous thermoreceptors may be an important factor in the initiation of continuous breathing at birth. Maintenance of a patent airway is also important in the continuation of effective ventilation after birth, but whereas in the adult the principal pharyngeal dilator is the genioglossus muscle, in the fetus genioglossus phasic activity is not synchronous with that of the fetal diaphragm. To ascertain whether a cold stimulus that initiates continuous breathing would also induce synchronized inspiratory activity in the upper airway muscles of the fetus, we have cooled fetal lambs in utero and examined the response of the genioglossus and alae nasi muscles in relation to diaphragm activity. Deep regular breathing as recorded by diaphragmatic activity and tracheal pressure started within 1-102 s after cooling commenced and coordinated inspiratory activity was seen in the alae nasi within 2-356 s. Genioglossus activity became synchronized with that of the diaphragm within 11-356 s. Cooling was continued for $4 \mathrm{~h}$ but although in one fetus continuous breathing with associated inspiratory activity in the genioglossus and alae nasi muscles lasted throughout the 4-h period, in the others continuous breathing was not sustained and adaptation to the cold stimulus occurred after periods ranging from $27-218 \mathrm{~min}$. The relationship between breathing, upper airway muscle activity, and sleep state passed through one or more different phases, including breathing through high voltage electrocortical activity, before the normal fetal pattern of episodic breathing restricted to the low voltage electrocortical state resumed. As breathing reverted to the normal fetal pattern, inspiratory activity was lost in the genioglossus. These observations suggest that afferent input arising from cutaneous thermoreceptors may be an important factor in initiating respiration at birth and in ensuring coordinated activity of the upper and lower airway and reducing the possibility of airway obstruction in the neonate. (Pediatr Res 23: 257261, 1988)
\end{abstract}

\section{Abbreviations}

ECOG, electrocorticogram

EMG, electromyogram

$\mathrm{HV}$, high voltage

$\mathrm{LV}$, low voltage

REM, rapid eye movement

Received June 25, 1987; accepted October 27, 1987.

Correspondence Dr. B. M. Johnston, Developmental Physiology Laboratory, Department of Paediatrics, School of Medicine, University of Auckland, Private Bag, Auckland 1, New Zealand.

Supported by grants from the Medical Research Council of New Zealand, the Wellcome Trust, and the National Children's Health Research Foundation (Cot Death Division)
The transition from fetus to neonate involves many physiological changes at birth and one of the most critical is the change from the fetal pattern of episodic breathing (1) to the continuous breathing necessary for the neonate's survival. We have suggested previously that cold stimulation of cutaneous thermoreceptors is an important factor in the initiation of continuous breathing at birth because rapid cooling achieved by passing cold water through a plastic coil placed around the fetal chest in utero causes the immediate onset of deep regular continuous breathing (2).

Another important factor in effective ventilation after birth is the maintenance of a patent airway. In the adult the principal pharyngeal dilator is the genioglossus muscle of the tongue and the nasal airway dilators are the alae nasi muscles. Although both these muscles function as inspiratory muscles in adult humans and rabbits $(3,4)$, in the late gestation fetal lamb only the alae nasi muscles show inspiratory activity (5). Whereas the alae nasi respond appropriately to hypercapnia and hypoxia, the genioglossus muscles do not show phasic activity synchronized with the diaphragm or alae nasi during periods of normal unstimulated fetal breathing, and even in conditions of extreme hypercapnia it is difficult to develop and maintain coordinated inspiratory activity in the genioglossus muscles (5).

Inasmuch as cooling in utero initiates deep regular breathing we wondered whether the cold stimulus might also induce coordinated inspiratory activity in the genioglossus. We were also interested in whether a more prolonged cooling period than the 60 min previously studied (2) would induce any permanent changes in the relationship between breathing and sleep state such as occur after birth. We have therefore studied the effect of $4-6 \mathrm{~h}$ of cooling on diaphragm, genioglossus, and alae nasi activity in fetal lambs of 125-140 days gestation.

\section{METHODS}

Surgical procedures. Using sterile techniques, operations were performed under halothane anaesthesia on eight pregnant Romney ewes at 120-135 days gestation. Polyvinyl catheters were implanted into the fetal carotid artery, jugular vein, trachea, and amniotic sac as well as into the maternal femoral artery and tarsal vein. Pairs of stainless steel wire electrodes (Cooner Wire Co., Chatsworth, CA) were implanted as described previously onto the dura to record the ECOG and into the nuchal, alae nasi, submental genioglossus muscles, and the diaphragm (5). In addition, thermistors (sensitive to $\pm 0.01^{\circ} \mathrm{C}$ ) were placed in the fetal esophagus, the amniotic fluid, and the maternal inferior vena cava. An "external" cooling coil consisting of $5 \mathrm{~m}$ blood warming coil (McGaw v45 20) was placed around the fetal neck and thorax (6). The coil was fixed by two or three sutures to the skin and the afferent and efferent tubing was exteriorised through the maternal flank along with the catheters, electrodes, and thermistors. After the operation the ewe was housed in a meta- 
bolic cage at constant temperature $\left(20^{\circ} \mathrm{C}\right)$ and humidity $(50 \%)$ and given free access to hay and water.

Experimental procedures. Recordings of ECOG, integrated nuchal, alae nasi, genioglossus, and diaphragm EMG together with tracheal and arterial pressures were started 6-24 h after the operation and continued for 10-14 days after which time the fetuses were sacrificed or assigned to other experiments which were not part of this study. The EMG were filtered (80-10000 $\mathrm{Hz}$ ) and passed through a leaky integrator with a time constant of 20 or $50 \mathrm{~ms}$. Antibiotics (80 mg gentamycin intramuscularly) were administered for 5 days postoperatively and experiments were not started until the 4th or 5th postoperative day and were carried out only if the $\mathrm{PO}_{2}$ was more than $20 \mathrm{~mm} \mathrm{Hg}$ and the $\mathrm{pH}$ greater than 7.30 .

Fetal and maternal temperature measurements were made by connecting the thermistors to a multichannel microprocessor controlled amplifier-transducer unit (17) and, starting $1 \mathrm{~h}$ before each experiment, temperatures were printed out at 1 -min intervals throughout the whole cooling period. The fetus was cooled for $4 \mathrm{~h}$ as described previously (2). Cold tap water $\left(14-20^{\circ} \mathrm{C}\right.$ ) was passed through the coil at rates varying from $100-300 \mathrm{ml} /$ min. A slower rate was usually used initially and this was increased if breathing stopped. If necessary, the degree of cold stimulation was increased by adding another $2-3 \mathrm{~m}$ of coil to the afferent limb of the fetal coil. This was then placed in a bucket of ice and water so that the tap water was cooled further before entering the amniotic sac.

Most polygraphic recordings were made with the paper running at a slow speed $(5 \mathrm{~mm} / \mathrm{min})$, but when a second polygraph was available we also displayed up to four channels of integrated and/or unprocessed EMG run at faster paper speeds (10-25 $\mathrm{mm} / \mathrm{s}$ ) periodically through the experiment. Arterial samples of $0.5 \mathrm{ml}$ were taken at hourly intervals for blood gas and $\mathrm{pH}$ analysis (Radiometer, Copenhagen, Denmark) and the values were corrected to the body temperature of the fetus by the factors shown to be appropriate for adult sheep blood (7).

These procedures were approved by the Animal Ethical Committee of the University of Auckland. All results are presented as mean $\pm \mathrm{SEM}$.

\section{RESULTS}

Fifteen experiments were carried out on a total of eight fetuses. In the control periods diaphragmatic activity was restricted to the epochs of LV ECOG activity. Similarly as described previously (5) phasic alae nasi activity was present through much of the LV state often coordinated with that of the diaphragm. Phasic genioglossus activity was also recorded only during the LV state but was not synchronous with, or coordinated to, diaphragmatic activity. As described previously (2), deep, regular breathing commenced within 1-102 s and the amplitude of the ECOG increased to show HV activity. Inspiratory activity was seen in the alae nasi within 2-356 s of the start of cooling and phasic genioglossus activity became synchronized with that of the diaphragm and alae nasi within 11-356 s. Figure 1 shows the change in the pattern of activity in the diaphragm, genioglossus, and alae nasi muscles between the control period and 2 min after cooling started. As cooling continued the activity of the genioglossus and the alae nasi muscles in relation to diaphragm and ECOG showed several different patterns. These patterns have been designated phases one to four as follows.

Phase one. The amplitude of the ECOG was higher that that seen during normal LV activity but was not always similar to normal HV activity although prolonged bursts of tonic activity were seen in the nuchal muscles. Breathing was deep and regular and synchronized inspiratory activity was seen in the alae nasi and genioglossus (Fig. 2A).

Phase two. Alternating periods of $\mathrm{HV}$ and LV ECOG were clearly distinguishable but respiratory activity was continuous through both states (Fig. 2B). Breathing associated with $\mathrm{HV}$ activity was usually deeper than that associated with LV activity although in both states it was much more regular than normal fetal breathing (30-40/min). Genioglossus and alae nasi activity was synchronous with the diaphragm during HV (similar to that seen in Fig. $1 B$ ), but during LV episodes activity in these muscles either disappeared or in the case of the genioglossus was not related to diaphragmatic activity. Alae nasi activity, if present, did show some coordination with the diaphragm during phase two, but this was not consistent.

Phase three. This phase was characterized by HV episodes in which breathing movements were not sustained or fell markedly to rates of only $7-10 / \mathrm{min}$ (Fig. 2C). Coordinated inspiratory activity in the genioglossus and alae nasi was usually present in association with breathing during $\mathrm{HV}$; during LV episodes there was often little activity in the genioglossus or alae nasi although breathing movements as reflected by the diaphragmatic and tracheal pressure recordings were present. Activity in the nuchal muscles indicative of shivering, disappeared entirely during LV episodes by this stage. Breathing could usually be restarted during the HV episodes by increasing the cold stimulation either by increasing the flow rate or by further cooling of the water flowing through the coil.

Phase four. This phase showed an essentially normal fetal pattern in which there was no breathing, alae nasi, or genioglossus activity in $\mathrm{HV}$ episodes, but breathing was present during LV ECOG (Fig. 2D). Phasic genioglossus activity was seen only during LV ECOG but was not coordinated with diaphragmatic activity. Increasing the degree of cold stimulation during this phase did not cause a return to breathing in HV ECOG.

Although all fetuses showed the pattern of breathing and ECOG characteristic of phase one, the duration of this phase varied from $27-240 \mathrm{~min}$ and after that not each of phases two to four was necessarily seen. Table 1 shows the duration of each phase for each experiment. On four occasions in three fetuses a pattern of positive tracheal pressure bursts was seen intermittently during $\mathrm{HV}$ episodes in phases three and four (Fig. 3) usually when there was little evidence of normal breathing movements. Closer examination revealed that this activity was associated with diaphragm activity of reduced amplitude and longer duration than was associated with inspiration. In addition, similar bursts of activity occurred simultaneously in the nuchal muscles suggestive of vigorous shivering.

Blood gas analysis showed a fall in $\mathrm{PO}_{2}$ and $\mathrm{pH}$ after $60 \mathrm{~min}$ as described previously (6) and the $\mathrm{PO}_{2}$ and $\mathrm{pH}$ remained low at 120,180 , and $240 \mathrm{~min}$ of cooling (Table 2). After cooling was stopped breathing continued for up to $30 \mathrm{~min}$ and then after a period of reduced breathing movements, the normal pattern was gradually reestablished overnight with no indication of any prolonged or permanent effect on breathing or ECOG.

\section{DISCUSSION}

We have previously shown that although the alae nasi muscles readily show inspiratory activity coordinated with that of the diaphragm, the genioglossus muscles in the fetal lamb do not normally behave as respiratory muscles and indeed coordinated inspiratory activity is difficult to induce even when fetal breathing movements are greatly stimulated by the administration of $10 \%$ $\mathrm{CO}_{2}$ to the ewe (5). Herein we demonstrate that cutaneous cold stimulation rapidly induces synchronization of inspiratory activity between the upper and lower airway. Our findings therefore suggest that the neural mechanisms responsible for controlling the coordination of genioglossus and diaphragm activity are present in late gestation; however, under normal conditions in utero such mechanisms are not operative. Failure of such synchronization to be initiated at and maintained after birth could be one mechanism by which primary obstructive apnea may be induced, although recent observations have shown that in newborn and adult sheep, during wakefulness at least, the genioglossus does not show inspiratory activity (19). The possible role of 

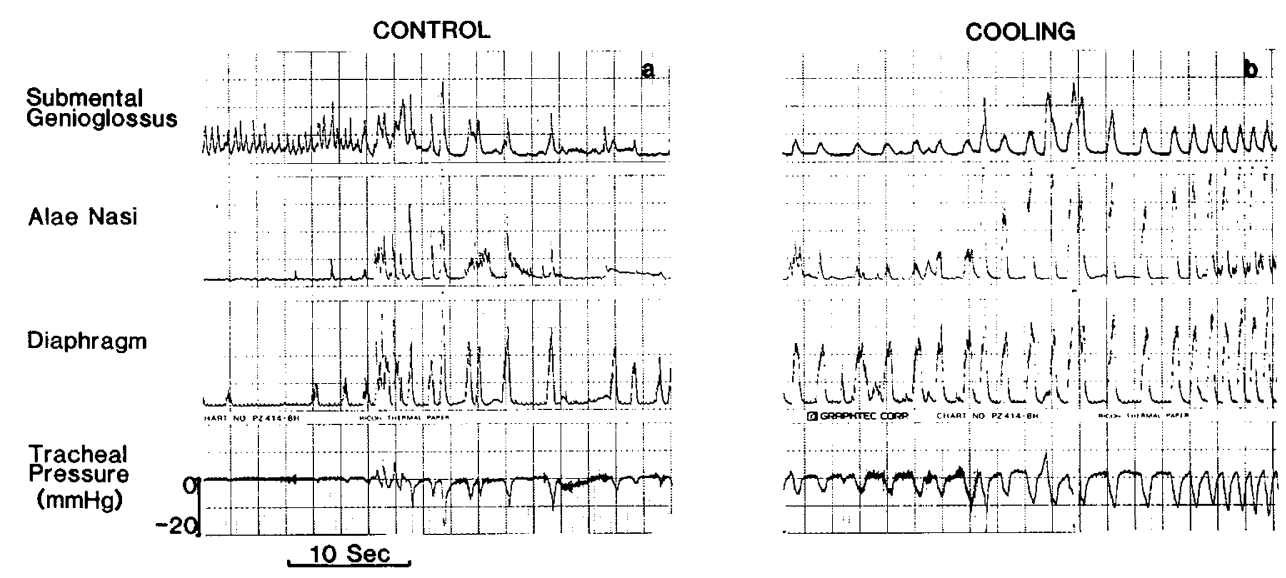

Fig. 1. Fast record of integrated genioglossus, alae nasi, diaphragm EMG, and tracheal pressure immediately before $(a)$ and 2 min after $(b)$ the start of cooling. Before cooling starts phasic activity in the genioglossus is not coordinated with that of the diaphragm. This is characteristic of low voltage ECOG. After cooling starts $(b)$ phasic activity in the genioglossus and alae nasi is rapidly coordinated with that of the diaphragm.
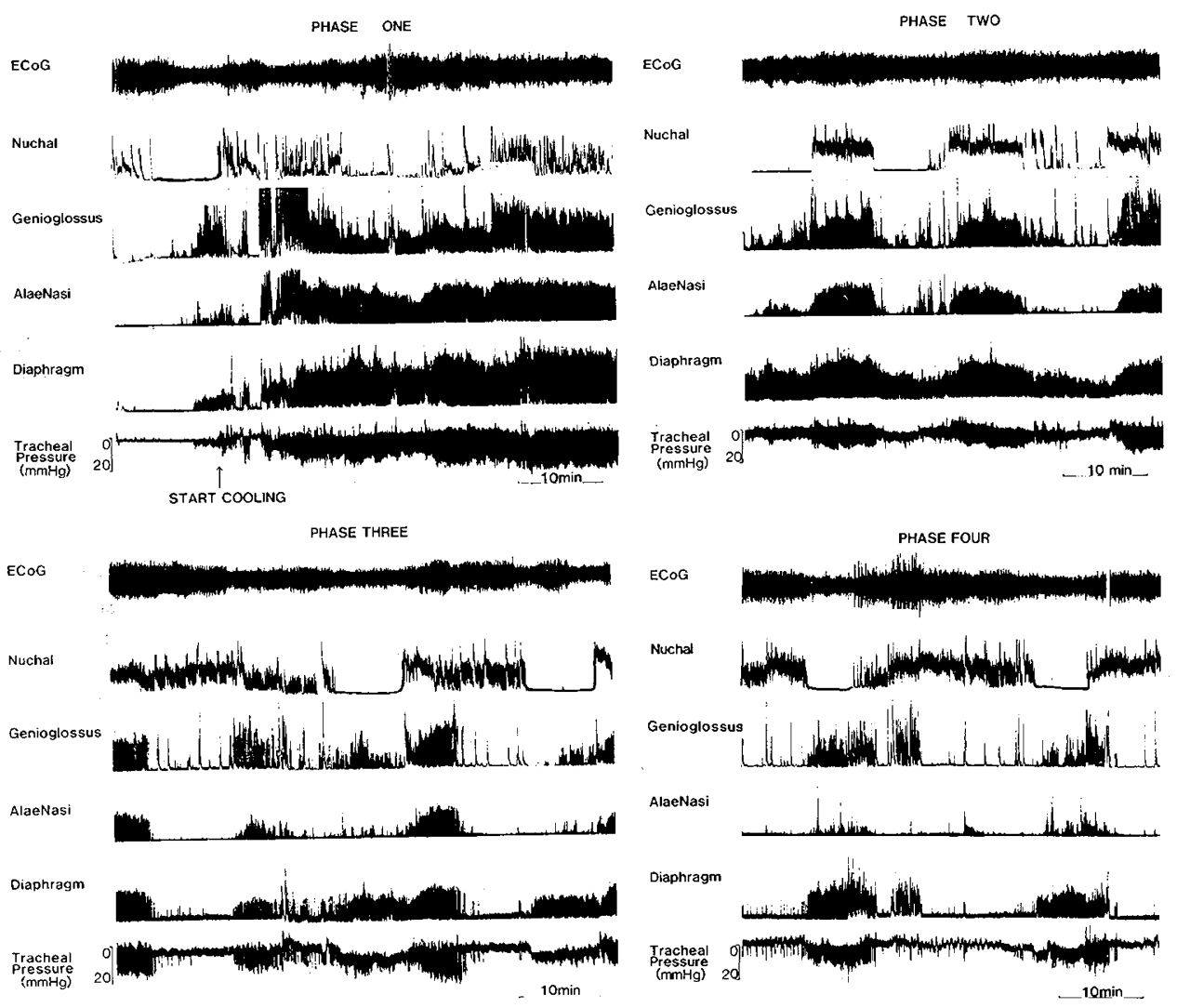

Fig. 2. Representative record of the relationship between ECOG, nuchal, genioglossus, alae nasi, diaphragm, and tracheal pressure in phases one to four during cooling. The record is run at a slow speed $(5 \mathrm{~mm} / \mathrm{min})$ to clarify the overall pattern of the relationship between sleep state and activity in the various muscles. In phase one activity in all the muscles is coordinated after cooling starts, whereas in phase two genioglossus and alae nasi activity are coordinated with the diaphragm only during HV ECOG. During phase two genioglossus activity either disappears or is not related to diaphragm activity. In phase three breathing movements are not sustained throughout the whole of the HV ECOG periods but when present the genioglossus, alae nasi, and diaphragm are coordinated. In LV ECOG breathing movements are present throughout but the genioglossus is not coordinated with the diaphragm. Phase four is similar to normal fetal breathing, i.e. in HV ECOG breathing movements are not present and there is little phasic genioglossus or alae nasi activity. Phasic genioglossus and alae nasi activity is seen in LV ECOG but the genioglossus is not coordinated with the diaphragm and the alae nasi is not consistently coordinated.

temperature in the genesis of obstructive apnea in the neonate requires further consideration.

In contrast to the resistance to other respiratory stimuli such as $\mathrm{CO}_{2}$, the rapidity with which the genioglossus developed a coordinated respiratory rhythm when the fetus was cooled is striking as the fetal core temperature had not begun to fall. A similar response has been seen in newborn and adult sheep where inspiratory genioglossus activity developed rapidly in response to nasal occlusion (19). It has recently been suggested that thermal factors are more important than chemoreceptor afferents in the control of breathing in the early neonatal period in the lamb (8). Our observations that breathing movements in the fetus are more readily induced by a cold stimulus (2) than by $\mathrm{CO}_{2}$, particularly in the high voltage state (9), and the present 
study showing that cold stimulation of cutaneous thermoreceptors induces coordination of upper and lower airways are further evidence for the central role of thermal input in the initiation and maintenance of effective respiration at birth.

The changing pattern of genioglossus and alae nasi activity and its relationship with sleep state during the cooling period is interesting because it is apparent that cold stimulation of cutaneous thermoreceptors in the fetus can cause both breathing and upper airway activity to be influenced by sleep state in a manner similar to that observed in adult species. Normally in the fetus, although sleep state affects both breathing and upper airway activity, its influence differs from that of the adult in that breathing, genioglossus, and alae nasi activity occur only in the LV state and genioglossus activity is not related to inspiration (5). During cooling the development of coordinated inspiratory activity in the genioglossus and alae nasi in association with the deep regular breathing present during the high voltage-like electrocortical activity of phases one and two is similar to that observed in adult man and rats in quiet sleep $(3,11,12)$. Similarly the pattern seen in phase two when the LV REM-like state reappears resembles the adult pattern where phasic inspiratory genioglossus or alae nasi activity is greatly reduced or absent in REM sleep and genioglossus or alae nasi activity, if present, is related to facial twitching or jaw movements rather than breathing $(3,11,13,14)$. A similar pattern has also been observed in

Table 1. Duration (min) that individual fetuses spent in each phase during cooling

\begin{tabular}{ccrrrr}
\hline Fetus & $\begin{array}{c}\text { Gestational } \\
\text { age (days) }\end{array}$ & $\begin{array}{c}\text { Phase } \\
\text { one }\end{array}$ & $\begin{array}{c}\text { Phase } \\
\text { two }\end{array}$ & $\begin{array}{c}\text { Phase } \\
\text { three }\end{array}$ & $\begin{array}{c}\text { Phase } \\
\text { four }\end{array}$ \\
\hline 1 & 130 & 38 & 202 & & \\
1 & 133 & 78 & 72 & 146 & 64 \\
2 & 133 & 27 & & 213 & \\
2 & 136 & 47 & & 193 & \\
3 & 128 & 79 & 37 & & 124 \\
3 & 131 & 122 & & 118 & \\
3 & 133 & 62 & 67 & & 111 \\
4 & 132 & 64 & & 40 & 136 \\
4 & 138 & 160 & 80 & & \\
5 & 126 & 218 & & 22 & \\
6 & 140 & 58 & & 182 & \\
7 & 131 & 240 & & & \\
7 & 132 & 86 & & 154 & \\
8 & 132 & 69 & & 171 & \\
8 & 133 & 134 & 28 & & 78 \\
\hline
\end{tabular}

preterm infants (15) and during wakefulness in newborn and adult sheep (19). It is also possible that phase one represents a state of arousal or wakefulness. Immersion of the fetal lamb in a cool bath has been shown to induce a change in sleep state with a rapid transition to wakefulness associated with the onset of breathing movements (18). However, the adult EEG in the awake state shows LV activity whereas in our fetuses the amplitude of the ECOG was high.

The observation that continuous breathing was not always maintained throughout the whole 4-h cooling period suggests that a certain amount of adaptation to the cold stimulus must occur. In addition the fall in $\mathrm{PO}_{2}$ and $\mathrm{pH}$ would act as an inhibitory influence on breathing $(2,10)$. We have previously suggested that the rate of change of stimulation of cutaneous thermoreceptors is important (2) and our findings are in agreement. They also show that breathing can be restarted to a certain degree by increasing the cold stimulus if adaptation has occurred. However, there are limits to the degree of cold stress that the fetus can withstand for prolonged periods and we did not continue increasing the cold stimulus once the fetal temperature had fallen by $3-4^{\circ} \mathrm{C}$.

It would be tempting to conclude that cold stimulation of the skin causes the switch from the fetal pattern of breathing and upper airway activity to the adult pattern, but this would be over simplifying because breathing is not always sustained throughout the whole cooling period. Clearly other factors must be important. It should also be pointed out that the clear modulation by sleep state of depth and rate of breathing seen in phase two has also been seen previously during infusion of certain drugs such as indomethacin (16). It would be interesting to know if genioglossus and alae nasi activity were also affected in the same way. Nevertheless it is clear that a cold stimulus to the cutaneous thermoreceptors exerts a powerful influence over the mechanisms coordinating breathing and upper airway activity and as soon as adaptation occurs and breathing ceases to be stimulated

Table 2. Fetal blood gases and temperature during cooling (mean $\pm S E M, n=14$ )

\begin{tabular}{ccccc}
\hline & $\mathrm{pH}$ & $\mathrm{PCO}_{2}$ & $\mathrm{pO}_{2}$ & Temperature ${ }^{\circ} \mathrm{C}$ \\
\hline 0 & $7.35 \pm 0.01$ & $46.4 \pm 2.0$ & $23.0 \pm 1.1$ & $39.76 \pm 0.08$ \\
$60^{\prime}$ & $7.30 \pm 0.01$ & $43.7 \pm 1.7$ & $15.5 \pm 0.8$ & $36.16 \pm 0.29$ \\
$120^{\prime}$ & $7.28 \pm 0.02$ & $46.0 \pm 2.0$ & $15.5 \pm 0.8$ & $35.64 \pm 0.26$ \\
$180^{\prime}$ & $7.28 \pm 0.02$ & $45.2 \pm 1.3$ & $14.2 \pm 0.9$ & $35.62 \pm 0.27$ \\
$240^{\prime}$ & $7.28 \pm 0.01$ & $47.2 \pm 1.2$ & $14.9 \pm 0.9$ & $35.59 \pm 0.22$ \\
\hline
\end{tabular}

Genioglossus

\section{Diaphragm}
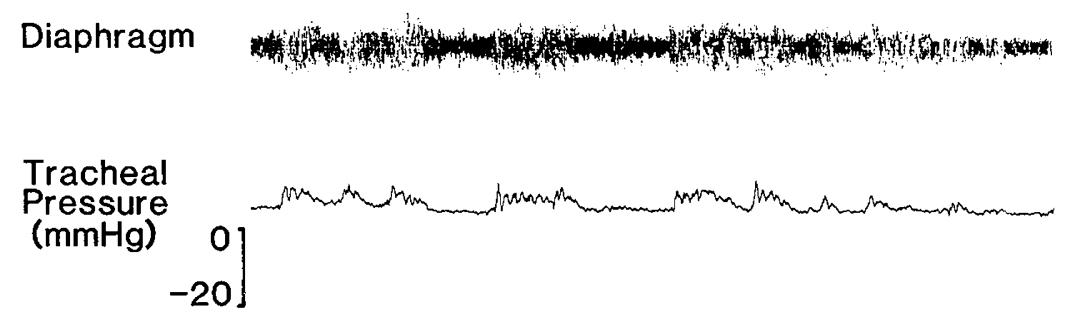

Nuchal

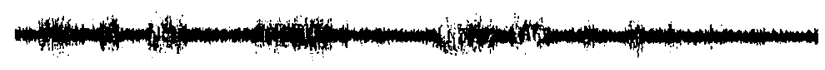

$5 \mathrm{sec}$

Fig. 3. Example of the positive tracheal pressure bursts seen during the later stages of cooling in three fetuses. These bursts occur in association with prolonged diaphragm and nuchal activity and are thus thought to be nonrespiratory in origin but instead due to vigorous shivering. 
and reverts back to the fetal pattern (phases three and four) so too does genioglossus and alae nasi activity.

\section{REFERENCES}

1. Dawes GS, Fox HE, Leduc BM, Liggins GC, Richards RT 1972 Respiratory movements and rapid eye movement sleep in the fetal lamb. J Physio 220:119-143

2. Gluckman PD, Gunn TR, Johnston BM 1983 The effect of cooling on breathing and shivering in unanaesthetized fetal lambs in utero. $\mathbf{J}$ Physiol 343:495-506

3. Sauerland EK, Harper RM 1976 The human tongue during sleep: electromyographic activity of the genioglossus muscle. Exp Neurol 51:160-170

4. Brouillette RT, Thach BT 1980 Control of genioglossus muscle inspiratory activity. J Appl Physiol 50:693-700

5. Johnston BM, Gunn TR, Gluckman PD 1986 Genioglossus and alae nasi activity in fetal sheep. $\mathrm{J}$ Dev Physiol 8:323-331

6. Gunn TR, Gluckman PD 1983 Development of temperature regulation in the fetal lamb. J Dev Physiol 5:167-179

7. Hales JRS, Bligh J, Maskrey M 1970 Cerebrospinal fluid acid-base balance during respiratory alkalosis in the panting animal. Am J Physiol 219:469 473

8. Johnson P 1986 Non-chemical factors in postnatal respiratory control and some of their clinical implications. In: Johnston BM, Gluckman PD (eds). Respiratory Control and Lung Development in the Fetus and Newborn. Perinatology Press, Ithaca, NY pp 377-395

9. Dawes GS, Gardner WN, Johnston BM, Walker DW 1982 Effects of hypercapnia on tracheal pressure, diaphragm and intercostal electromyograms in unanaesthetized fetal lambs. J Physiol 326:464-474

10. Boddy K, Dawes GS, Fisher RL, Pinter S, Robinson JS 1974 Foetal respiratory movements, electrocortical and cardiovascular responses to hypoxaemia and hypercapnia in sheep. J Physiol 243:599-618

11. Remmers JE, de Groot WJ, Sauerland EK, Anch AM 1978 Pathogenesis of upper airway occlusion during sleep. J Appl Physiol 44:931-938

12. Megirian D, Hinrichsen CFL, Sherrey JH 1985 Respiratory roles of genioglossus sternothyroid and sternohyoid muscles during sleep. Exp Neurol 90:118128

13. Megirian D, Cespuglio R, Jouvet M 1978 Rhythmical activity of the rat's tongue in sleep and wakefulness. Electroencephalogr Clin Neurophysiol 44:8-13

14. Sherrey JH, Megirian D 1977 State dependence of upper airway respiratory motorneurons: Functions of the cricothyroid and nasolabial muscles of the unanaesthetized rat. Electroencephalogr Clin Neurophysiol 83:318-228

15. Carlo WA, Martin RJ, Abboud ER, Bruce EN, Strohl KP 1983 Effect of sleep state and hypercapnia on alae nasi and diaphragm EMGs in preterm infants. J Appl Physiol 54:1590-1596

16. Kitterman JA, Liggins GC, Clements JA, Tooley WH 1980 Stimulation of breathing movements in fetal sheep by inhibitors of prostaglandin synthesis. J Dev Physiol 453-466

17. Gluckman PD, Gunn TR, Johnston BM, Quinn JP 1984 Manipulation of the temperature of the fetal lamb in utero. In: Nathanielsz PW (ed) Animal Models in Fetal Physiology, III. Perinatology Press, Ithaca, NY, pp 37-56

18. Moss IR, Mautone AJ, Scarpelli EM 1983 Effect of temperature on regulation of breathing and sleep/wake state in fetal lambs. J Appl Physiol 54:536-543

19. Harding R, Buttress JA, Caddy DJ, Wond GA 1987 Respiratory and upper airway responses to nasal obstruction in awake lambs and ewes. Respir Physiol 68:177-188 